\title{
Emulsion Stability-Theoretical Studies on Simultaneous Flocculation and Creaming
}

\author{
S. R. REDDY, ${ }^{1}$ D. H. MELIK, AND H. S. FOGLER \\ Department of Chemical Engineering, University of Michigan, Ann Arbor, Michigan 48109
}

Received April 16, 1980; accepted October 30, 1980

\begin{abstract}
A general dynamic equation is derived describing the behavior of a polydispersed emulsion in which Brownian flocculation, sedimentation-flocculation, and creaming are taking place simultaneously. The resulting equation consists of a set of coupled partial differential equations, which are solved numerically to predict changes in particle concentration and size distribution as a function of time and position. Predictions are also made for various limiting cases, such as negligible creaming, negligible flocculation, and for various degrees of electrostatic stabilization. A cyclic change in particle size distribution is observed when flocculation and creaming occur simultaneously.
\end{abstract}

\section{INTRODUCTION}

The general problem of emulsion stability with simultaneous flocculation and creaming has not yet been solved to predict the behavior of polydisperse emulsions. Simultaneous flocculation and creaming is a very complex process in which emulsion particles under Brownian motion are colliding to form larger particles. At the same time, particles are creaming out owing to the difference in the densities of particles and the medium. In addition to these two processes there is a third coupled process called sedimentation-flocculation. This emulsionbreaking mechanism is due to the different creaming rates of different size particles. Since larger particles cream out at a faster rate than the smaller ones, collisions of the larger particles with the slower moving smaller particles occur as they cream out. In most common emulsions all these processes are taking place at the same time, equally significant, and inseparable. To characterize the behavior of the emulsion, it is essential that all three emulsion-

\footnotetext{
${ }^{1}$ Present Address: General Motors Research Laboratories Warren, Michigan 48090.
}

breaking mechanisms, flocculation, creaming, and sedimentation-flocculation be considered.

The purpose of this study is to describe this complex emulsion stability. This problem is encountered in many practical emulsions; e.g., stability of water-in-fuel emulsions. Water-in-fuel emulsions are considered for use as fuel extenders (1) which can be prepared ultrasonically with particles in the submicron size range (2). The particles flocculate and form larger particles and cream out to the top. This takes place very rapidly and water and fuel separate within a few minutes. It is not possible to predict the behavior of such emulsions by solving either the flocculation or the creaming problem alone. This problem is also encountered in enhanced oil recovery $(3,4)$. Demulsification of crude oil-in-water involves simultaneous flocculation and creaming. Wasan et al. (4) studied these two processes separately, coalescence of droplets and creaming rate of dispersed phase.

Investigators $(5,6)$ in the aerosol field have studied an approximate and simplified problem involving flocculation and settling of aerosol particles. Okuyama (7) studied 
the flocculation and sedimentation problem by assuming that the particle size distribution is spatially uniform, and without including sedimentation-flocculation. The behavior of emulsion particles is quite different from that of aerosol particles, owing to the fact that emulsion particles are mostly electrostatically stabilized and the stability increases exponentially with increasing particle size. Changes in particle concentration and particle size distribution are a strong function of electrostatic stability. The changes in emulsion particle size distributions will be quite different from aerosol particles. The object of this study is to predict the changes in local particle size and size distribution as a function of time and position in an emulsion in which flocculation, creaming, and sedimentation-flocculation are taking place simultaneously.

\section{THEORY}

The general equation describing emulsion stability is derived by performing a population balance on the emulsion particles. The resulting equation, which includes Brownian flocculation, sedimentation-flocculation, and creaming, is a nonlinear, partial integrodifferential equation which cannot be solved analytically. The general equation will be modified so that it can be solved numerically. It will be assumed that particle concentration varies only in the direction of the gravitational field and coalescence occurs as soon as the particles flocculate. Initially, the emulsion is spatially uniform, with a wide range of particle sizes. Since thermal gradients are negligible, there are no convective currents in the system.

The basis of the general stability equation is a particle balance over any size range $\nu$ to $\nu+d \nu$ in the elemental volume, $\Delta V$, of emulsion at a distance, $y$, and time, $t$. Particles in the size range $\nu$ to $\nu+d \nu$ enter into and leave $\Delta V$ by creaming. In addition, particles in this size range appear and disappear by flocculation with other size particles.

The Brownian collision frequency between particles of size $\nu$ to $\nu+d \nu$ and $\tilde{\nu}$ to $\tilde{\nu}+d \tilde{\nu}$ is given by the expression $(5,8)$

$$
B(\nu, \tilde{\nu}) n(\nu, y, t) n(\tilde{\nu}, y, t) d \nu d \tilde{\nu} .
$$

The gravitational collision frequency for the same particle sizes is

$$
G(\nu, \tilde{\nu}) n(\nu, y, t) n(\tilde{\nu}, y, t) d \nu d \tilde{\nu} .
$$

The Brownian collision frequency function $B(\nu, \tilde{\nu})$ is given by the Stokes-Einstein relation (5)

$$
B(\nu, \tilde{\nu})=\frac{2 k_{\mathrm{b}} T}{3 \mu W}\left(\frac{1}{\nu^{1 / 3}}+\frac{1}{\tilde{\nu}^{1 / 3}}\right)\left(\nu^{1 / 3}+\tilde{\nu}^{1 / 3}\right) .
$$

Assuming the particle paths are straight vertical lines, the collision efficiency is unity, and the stability factor is identical to that of the Brownian collision frequency; the gravitational collision frequency function, $G(\nu, \tilde{\nu})$, is given by $(5,8,9)$

$$
G(\nu, \tilde{\nu})=\frac{2 \pi \Delta \rho g}{9 \mu W}\left|r^{2}-\tilde{r}^{2}\right|(r+\tilde{r})^{2},
$$

where

$$
\begin{aligned}
n(\nu, y, t) d \nu= & \text { concentration of particles } \\
& \text { with volume between } \nu \text { and } \\
\nu+d \nu \text { at distance } y \text { and } & \\
& \text { time } t, \\
r, \tilde{r}= & \text { the particle radii correspond- } \\
& \text { ing to particle volumes } \nu \text { and } \\
& \bar{\nu}, \text { respectively, } \\
W= & \text { the stability factor which is a } \\
& \text { function of electrostatic prop- } \\
& \text { erties, } T, \mu, \text { and the size of } \\
& \text { the particles }(10), \\
T= & \text { temperature, }{ }^{\circ} \mathrm{K}, \\
\mu= & \text { the viscosity of the continu- } \\
& \text { ous medium, and } \\
k_{\mathrm{b}}= & \text { the Boltzmann constant. }
\end{aligned}
$$

The general population balance equation on an elemental height $\Delta y$ can be expressed in mathematical form as follows: 


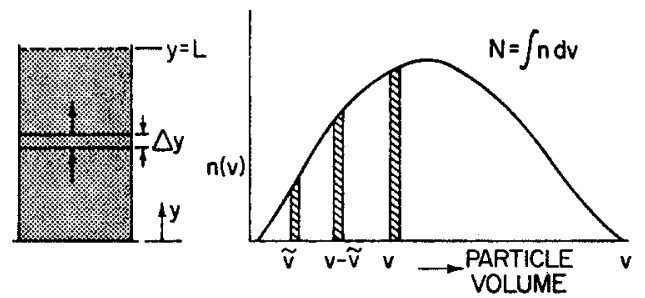

PARTICLE BALANCE OVER ANY SIZE RANGE $v$ to $v+d v$ IN THE ELEMENTAL VOLUME $\triangle V$ :

$$
\begin{aligned}
& \Delta v \frac{\partial[n(v, v, t) d v]}{\partial t}=\left.A \cdot u(v) \cdot n(v, y, t) d v\right|_{y}-\left.A \cdot u(v) \cdot n(v, y, t) d v\right|_{y}+\Delta y \\
& \begin{array}{l}
\text { ACCUMULATION ENTERING } \\
+A \cdot \Delta y \cdot \frac{1}{2} \int_{\tilde{v}=v_{\min }} B(\tilde{v}, v-\tilde{v}) n(\tilde{v}, y, t) n(v-\tilde{v}, y, t) d \tilde{v} d v
\end{array} \\
& v=v_{\min } \text { GAIN BY BROWNIAN FLOCCULATION } \\
& \ddot{v}=v_{\text {max }} \\
& -A \cdot \Delta y \int_{\tilde{v}=v_{\min }}^{\Delta} B(v, \widetilde{v}) n(v, y, t) n(\widetilde{v}, y, t) d \tilde{v} d v \\
& +A \cdot \Delta y \cdot \frac{1}{2} \int_{\tilde{v}=v-v_{\min }}^{\tilde{G}} G(\tilde{v}, v-\tilde{v}) \ln (\tilde{v}, y, t) n(v-\tilde{v}, y, t) d \tilde{v} d v \\
& \tilde{v}=v_{\min }
\end{aligned}
$$

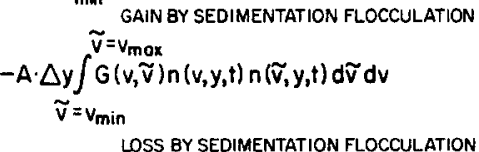

where

$A=$ cross-sectional area of the container, $U(\nu)=$ creaming velocity of particles with volume, $\nu$,

$\nu_{\min }=$ volume of the smallest particle in the emulsion, and

$\nu_{\max }=$ volume of the largest particle in the emulsion.

Dividing Eq. [1] by $A \times \Delta y$ and taking the limit as $\Delta y$ approaches zero results in:

$$
\begin{aligned}
& \frac{\partial n(\nu, y, t)}{\partial t}=-U(\nu) \frac{\partial n(\nu, y, t)}{\partial y} \\
& \quad+\frac{1}{2} \int_{\tilde{\nu}=\nu_{\min }}^{\tilde{\nu}=\nu-\nu_{\min }}[B(\tilde{\nu}, \nu-\tilde{\nu})+G(\tilde{\nu}, \nu-\tilde{\nu})] \\
& \quad \times n(\tilde{\nu}, y, t) n(\nu-\tilde{\nu}, y, t) d \tilde{\nu} \\
& \quad-\int_{\tilde{\nu}=\nu_{\min }}^{\tilde{\nu}=\nu_{\max }}[B(\nu, \tilde{\nu})+G(\nu, \tilde{\nu})] n(\nu, y, t)
\end{aligned}
$$$$
\times n(\tilde{\nu}, y, t) d \tilde{\nu} .
$$

Equation [2] is called the general dynamic equation $(5,6)$ for the continuous distribution function of particles. Since the general equation is already a nonlinear, partial integrodifferential equation, one can include other processes, such as laminar or turbulent shear induced flocculation, without increasing the complexity of the problem. A very general equation of this type is also called the population balance equation (11).

Since the general equation is a nonlinear, partial integrodifferential equation, its analytic solution is difficult. Analytical solutions had been obtained for very special cases of dynamical processes in aerosol behavior. Equation [2] can be solved numerically by representing the particles as a discrete distribution instead of a continuous distribution. Two methods which are used for relatively polydisperse systems are equal diameter incrementing with mass 
balance correction developed by Reddy (12) and the method of sectionalization proposed by Gelbard and Seinfeld (13). Even though both methods yield relatively similar results the sectionalization technique has advantages over the equal diameter-incrementing approach.

The solution to the set of coupled nonlinear partial differential equations using equal diameter incrementing gives the number concentration for each particle group $k$ as a function of time and position. The particle diameter of each group $k$ is a positive integer multiple of an arbitrarily chosen group 1 diameter. With this approach the collision of two particles seldom produces a particle with a diameter that matches a defined group diameter. That is, the particle's diameter lies between adjacent groups. In this case the particle is classified into the larger diameter group and a mass balance correction factor adjusts the particle number by adding only a fraction of a particle to this group (12). In the sectionalization method the particle size range is divided into sections where one can choose the sectional divisions based on any characteristic parameter that can be related to a particle's volume (e.g., diameter, log (diameter), volume, surface area, mass, etc.). The solution to the coupled sectionalization equa- tions gives the changes in a user-specified integral quantity for each section (e.g., particle number, volume, surface area) as a function of time and position. The integral quantity is conserved within each section and flocculation coefficients properly account for flocculations among all particle sizes. The computing time required for the sectionalization method is approximately $60 \%$ less than the equal diameter-incrementing technique.

In the study of simultaneous flocculation and creaming it is useful to follow changes in the number concentration for each section and to base the sectional increments on particle diameter or radius. For this case the number of particles in section $k$ is given by:

where:

$$
\begin{gathered}
N_{k}(y, t)=\int_{r_{k-1}}^{r_{k}} n(r, y, t) d r \\
k=1,2,3, \ldots, n, \\
r_{0}<r_{1}<r_{2}, \ldots,<r_{n},
\end{gathered}
$$

$n(r, y, t)=$ size distribution function,

$r=$ particle radius,

$y=$ particle position,

$t=$ time, and

$n=$ number of sections.

Using the sectionalization technique, Eq. [2] can be rewritten as

$$
\begin{aligned}
\frac{\partial N_{k}(y, t)}{\partial t}=- & \hat{U}_{k} \frac{\partial N_{k}(y, t)}{\partial y}+\frac{1}{2} \sum_{i=1}^{k-1} \sum_{j=1}^{k-1} 1 \bar{\beta}_{i, j, k} N_{i}(y, t) N_{j}(y, t)-N_{k}(y, t) \sum_{i=1}^{k-1}{ }^{2} \bar{\beta}_{i, k} N_{i}(y, t) \\
& -\frac{1}{2}{ }^{3} \bar{\beta}_{k, k} N_{k}^{2}(y, t)-N_{k}(y, t) \sum_{i=k+1}^{n}{ }^{4} \bar{\beta}_{i, k} N_{i}(y, t) \quad k=1,2,3, \ldots, n .
\end{aligned}
$$

The inter- and intrasectional flocculation coefficients, ${ }^{1} \bar{\beta}_{i, j, k},{ }^{2} \bar{\beta}_{i, k},{ }^{3} \bar{\beta}_{k, k}$, and ${ }^{4} \bar{\beta}_{i, k}$, are averaged collision frequencies which affect the net rate of addition or removal of particles from section $k$. The net rate of addition of particles into section $k$ is the result of all flocculations between particles in sections lower than section $k$. Particles of a given size can only be formed by the coalescence of smaller particles since mechanical breakage is absent. These coagulations are represented by the second term on the right-hand side (RHS) of Eq. [4]. The flocculation co- 
efficient is defined as:

$$
\begin{aligned}
& { }^{1} \bar{\beta}_{i, j, k}=\int_{r_{i-1}}^{r_{i}} \int_{r_{j-1}}^{r_{j}} \\
& \quad \times \frac{\theta\left(r_{k-1}^{3}<r^{3}+r^{3}<r_{k}^{3}\right) \beta(\tilde{r}, r)}{\left(r_{i}-r_{i-1}\right)\left(r_{j}-r_{j-1}\right)} d \tilde{r} d r,
\end{aligned}
$$

where:

$$
\begin{aligned}
& \beta(\tilde{r}, r)=B(\tilde{r}, r)+G(\tilde{r}, r), \\
& B(\tilde{r}, r)=(2 k T / 3 \mu W(\tilde{r}, r))(1 / \tilde{r}+1 / r)(\tilde{r}+r),
\end{aligned}
$$

and

$$
\begin{aligned}
G(\tilde{r}, r)=(2 \pi \Delta \rho g / 9 \mu W(\tilde{r}, r)) \mid & \tilde{r}^{2}-r^{2} \mid \\
& \times(\tilde{r}+r)^{2} .
\end{aligned}
$$

The net rate of removal of particles from section $k$ is the sum of the collision of particles in section $k$ and (1) particles in lower sections, (2) other particles in section $k$, and (3) particles in higher sections. These three processes are represented by the third, fourth, and fifth terms on the RHS of Eq. [4], respectively. The flocculation coefficients are given by

$$
\begin{aligned}
{ }^{2} \bar{\beta}_{i, k} & =\int_{r_{i-1}}^{r_{i}} \int_{r_{k-1}}^{r_{k}} \frac{\theta\left(\tilde{r}^{3}+r^{3}>r_{k}^{2}\right) \beta(\tilde{r}, r)}{\left(r_{i}-r_{i-1}\right)\left(r_{k}-r_{k-1}\right)} d \tilde{r} d r \\
{ }^{3} \bar{\beta}_{k, k} & =\int_{r_{k-1}}^{r_{k}} \int_{r_{k-1}}^{r_{k}} \frac{\left[2 \theta\left(\tilde{r}^{3}+r^{3}>r_{k}^{3}\right)+\theta\left(\tilde{r}^{3}+r^{3}<r_{k}^{3}\right)\right] \beta(\tilde{r}, r)}{\left(r_{k}-r_{k-1}\right)^{2}} d \tilde{r} d r \\
{ }^{4} \bar{\beta}_{i_{s k}} & =\int_{r_{i-1}}^{r_{i}} \int_{r_{k-1}}^{r_{k}} \frac{\beta(\tilde{r}, r)}{\left(r_{i}-r_{i-1}\right)\left(r_{k}-r_{k-1}\right)} d \tilde{r} d r .
\end{aligned}
$$

The discontinuous functions $\theta$ determine whether a collision between particles of radius $r$ and radius $\tilde{r}$ will result in the addition or removal of particles from section $k$. For example, if one considers particle collisions among all sections prior to section $k$ which might result in the addition of particles to section $k$ the flocculation coefficients are given by Eq. [5]. If a particle of radius $\tilde{r}$ from section $j$ collides with a particle of radius $r$ from section $i$ and the resulting particle's radius is between $r_{k-1}$ and $r_{k}$ there will be the addition of one particle to section $k$. If the particle's radius is either less than $r_{k-1}$ or greater than $r_{k}$, no new particle will be added to section $k$. For this example the $\Theta$ function takes the following form:

$$
\begin{aligned}
& \Theta\left(r_{k-1}^{3}<r^{3}+\tilde{r}^{3}<r_{k}^{3}\right) \\
& =1, \text { if } r_{k-1}^{3}<r^{3}+\tilde{r}^{3}<r_{k}^{3} \\
& =0, \text { if } r^{3}+\tilde{r}^{3}<r_{k-1}^{3} \text { or } r^{3}+\tilde{r}^{3}>r_{k}^{3} .
\end{aligned}
$$

In general the function $\Theta$ takes the following values:
$\Theta$ (condition)

$=1$, if the condition is satisfied

$=\mathbf{0}$, if the condition is not satisfied.

The particle-creaming velocity for section $k, \hat{U}_{k}$, can be calculated from Stoke's law, $U(r)$ :

$$
\begin{aligned}
U_{k} & =\frac{\int_{r_{k-1}}^{r_{k}} U(r) d r}{r_{k}-r_{k-1}}=\frac{2 g\left(\rho_{\mathrm{c}}-\rho_{\mathrm{s}}\right)}{9 \mu} \frac{\int_{r_{k-1}}^{r_{k}} r^{2} d r}{r_{k}-r_{k-1}} \\
& =\frac{2 g\left(\rho_{\mathrm{c}}-\rho_{\mathrm{s}}\right)}{27 \mu}\left[r_{k}^{2}+r_{k} r_{k-1}+r_{k-1}^{2}\right], \quad \text { [9] }
\end{aligned}
$$

where:

$$
g=\text { the gravitational constant, }
$$

$r_{k}=$ upper-bound particle radius in section $k$,

$r_{k-1}=$ lower-bound particle radius in section $k$, 
$\rho_{\mathrm{c}}=$ the density of the continuous medium, and

$\rho_{\mathrm{s}}=$ the density of the particle.

The stability factor, $W(\tilde{r}, r)$, for the interaction of two particles with radii $\tilde{r}$ and $r$ is given by the expression $(8,14)$ :

$$
\begin{aligned}
& W(\tilde{r}, r)=2 \int_{0}^{\infty} Z(\tilde{r}, r) \\
& \left.\times \exp \left[V_{\mathrm{T}}(\tilde{r}, r) / k_{\mathrm{b}} T\right)\right] \frac{d u}{(u+2)^{2}},
\end{aligned}
$$

where:

$V_{\mathrm{T}}(\tilde{r}, r)=$ the total interaction potential between particles of radii $\tilde{r}$ and $r$,

$Z(\tilde{r}, r)=$ hydrodynamic correction factor for particles of radii $\bar{r}$ and $r$, $u=2 H /(\tilde{r}+r)$, where $H$ is the shortest interparticle distance.

From the DLVO theory (15), the total interaction potential is given by the expressions:

for $H \leq 150 \AA$

$$
\begin{aligned}
V_{\mathrm{T}}(\tilde{r}, r)= & \frac{\tilde{r} r}{\tilde{r}+r}\left[\epsilon \psi^{2} \ln \left(1+e^{-K H}\right)\right. \\
& \left.-\frac{A}{6 H}\left(\frac{\lambda}{\lambda+11.116 H}\right)\right] .
\end{aligned}
$$

For $H>150 \AA$

$$
\begin{array}{r}
V_{\mathrm{T}}(\tilde{r}, r)=\frac{\tilde{r} r}{\tilde{r}+r}\left[\epsilon \psi^{2} \ln \left(1+e^{-K H}\right)\right. \\
-A\left(\frac{2.45 \lambda}{60 \pi H^{2}}-\frac{2.17 \lambda^{2}}{360 \pi^{2} H^{3}}\right. \\
\left.\left.-\frac{.59 \lambda^{3}}{1680 \pi^{3} H^{4}}\right)\right],
\end{array}
$$

where:

$$
\begin{aligned}
\epsilon= & \text { the dielectric constant of the medium, } \\
\psi= & \text { the surface potential, } \\
K= & \text { the reciprocal of the double layer } \\
& \text { thickness, } \\
\lambda= & \text { the characteristic wavelength of the } \\
& \text { atoms, and } \\
A= & \text { the Hamaker constant. }
\end{aligned}
$$

The hydrodynamic correction factor, which accounts for the viscous drag dependence on interparticle distance, is usually expressed as a series expansion (16). In order to facilitate numerical calculations an approximate function can be used for the hydrodynamic correction factor (16):

$$
Z(\tilde{r}, r)=\frac{6 s^{2}+13 s+2}{6 s^{2}+4 s},
$$

where

$$
s=H(\tilde{r}+r) / 2 \tilde{r} r
$$

\section{METHOD OF SOLUTION}

The behavior of an emulsion or the changes in emulsion particle concentration are described by the system of $n$ nonlinear coupled partial differential equations represented by general Eq. [4]. The solution to this system of equations gives the particle concentration for each section, $k$, as a function of time and position. Since an analytical solution is not possible, the equations have to be solved numerically.

The inter- and intrasectional flocculation coefficients (Eqns. 5, 6,7, and 8) were evaluated using a two-dimensional Simpson's rule algorithm (17). These coefficients need to be calculated only once and then stored. The system of $n$ nonlinear partial differential equations were solved using the semi-implicit Crank-Nicolson finite divided difference method (17). In using a finite difference technique the particle size distribution is assumed to be uniform at any given time within each segment $\Delta y$ and changes in the particle size distribution occur in steps $\Delta t$, for any given position. The magnitude of the time step $\Delta t$ is based on the stability factor, $W$, e.g., if the emulsion is very stable, changes in the particle size distribution will be slow, permitting a large time step. The size of the segment $\Delta y$ is based on the magnitude of the density difference, $\Delta \rho$. If $\Delta \rho$ is small, particles are creaming at a relatively slow rate and the change in particle concentration with 
a change in distance will be small allowing a relatively large space step, $\Delta y$, to be chosen. The concentration and size distributions in the first segment $\Delta y$ are denoted by $y=0$. Likewise, the concentration and size distributions in the last segment of thickness $\Delta y$ are specified by $y=L$.

The initial particle size distribution and concentration, electrostatic properties, and physical properties of the emulsion are required before starting the numerical computations. Since the stability factor, $W(\tilde{r}, r)$, needs to be evaluated several times, a reasonably accurate and economical numerical integration formula is desired. The following Gaussian quadrature formulas were chosen: The 9-point Gauss-Legendre quadrature for evaluating the integral from 0 to $b$, and the 9-point Gauss-Leguerre quadrature for $b$ to infinity.

$$
\begin{aligned}
& W(\tilde{r}, r)=2 \int_{0}^{b} Z(\tilde{r}, r) \exp \left[V_{\mathrm{T}}(\tilde{r}, r) / k_{\mathrm{b}} T\right] \\
& \times \frac{d u}{(u+2)^{2}}+2 \int_{b}^{\infty} Z(\tilde{r}, r) \\
& \quad \times \exp \left[V_{\mathrm{T}}(\tilde{r}, r) / k_{\mathrm{b}} T\right] \frac{d u}{(u+2)^{2}},
\end{aligned}
$$

where $b$ is chosen as twice the electric double layer thickness, i.e., $b=2 / K$.

\section{TABLE I}

\begin{tabular}{ll}
\hline Temperature & $78^{\circ} \mathrm{C}$ \\
Density of the continuous medium & $0.99 \mathrm{~g} / \mathrm{cm}^{3}$ \\
Density of dispersant & $0.77 \mathrm{~g} / \mathrm{cm}^{3}$ \\
Viscosity of the continuous medium & $0.37 \mathrm{cP}$ \\
Initial partical concentration & $9.0 \times 10^{9}$ \\
& part. $/ \mathrm{cm}^{3}$ \\
The 2-2 electrolyte concentration & $8.31 \times 10^{-3}$ \\
& $\mathrm{gmole} / 1$ iter \\
Zeta potential or surface potential, $\Psi$ & $10 \mathrm{mV}$ \\
Dielectric constant of the continuous & 61.0 \\
$\quad$ medium & $10^{-14} \mathrm{erg}$ \\
Hamaker constant (18) & $10^{-5} \mathrm{~cm}$ \\
Wavelength (19) &
\end{tabular}

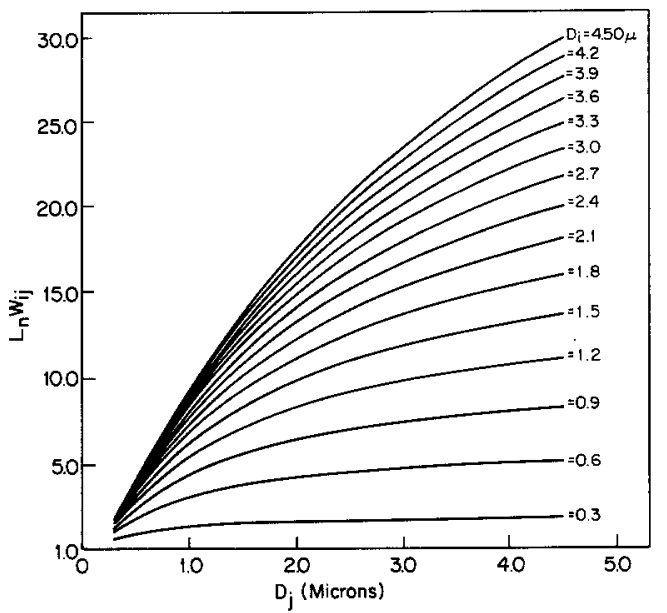

FIG. 1. Logarithm of stability factor, $W$, as a function of $D_{j}$ with parameter $D_{i}$.

\section{DISCUSSION}

The general equation for emulsion stability has been solved numerically for various initial particle concentrations and particle size distributions (PSD). In order to explore thoroughly the behavior of an emulsion in which flocculation and creaming are to occur simultaneously, it is necessary to choose the proper concentration and PSD. If particles are too small, creaming will be negligible and, if they are too large, floccuation will be negligible. The PSDs chosen are similar to those produced by acoustic emulsification. The same initial concentrations were used in all simulation studies. The initial particle size distribution can be found in any of the following PSD plots.

The physical and electrochemical properties used in the computations are shown in Table I and are similar to those for a straightchain hydrocarbon $\mathrm{C}_{36} \mathrm{H}_{74}$ emulsified in water.

In all of the following figures, particle size distributions are plotted as number density as a function of particle diameter in microns. Number density, $n(D)$, is related to distribution function, $P(D)$, as follows:

$$
n(D)=\frac{P(D)}{N}
$$




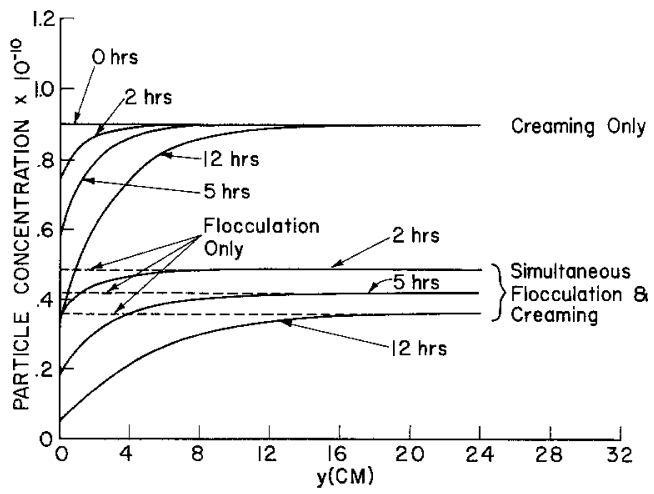

FIG. 2. Total particle concentration, $N$, as a function of time and position, $y$, for various cases.

and

$(P(D) /(N)) d D=$ fraction of particles

with diameters between $D$ and $D+d D$,

where $P(D)$ is particle size distribution function, and $N$ is the total number of particles of all sizes.

The computed stability factors are shown in Fig. 1. The stability factor increases exponentially with particle size. Smaller particles are unstable to flocculation, while larger particles are very stable.

The behavior of the emulsion was predicted by solving the set of partial differential equations numerically. For comparison, the behavior of the same emulsion was also predicted for the cases of only flocculation and only creaming. The changes in the particle concentration profiles can be seen in Fig. 2. If particles are only creaming to the top, a concentration profile develops with time with lower concentrations at the bottom of the column while the concentration at the top of the column remains virtually unchanged after $12 \mathrm{hr}$. If only flocculation is occurring, the particle concentration decreases uniformly throughout the column with time so that no spatial variations develop. In the case of flocculation and creaming, a profile develops as the concentration decreases. In the following figures, the changes in PSD are at the lower end of the column where the concentration profile is steep.

The changes in the PSD at the bottom of the column, $y=0$, as a function of aging time are shown in Fig. 3 for the case when only creaming is occurring ( $W \gg 1$ ). In this case, the PSD shifts to the left toward smaller size particles, since the larger particles cream out, increasing the relative concentration of smaller particles. Changes in the PSD when particles are only flocculating without creaming $(\Delta \rho=0)$ are presented in Fig. 4. Here, the emulsion particles are flocculating and forming larger particles, and consequently the PSD shifts to the right toward larger size particles. In this case, the PSD is the same everywhere in the column.

The changes in the PSD at the bottom of column, $y=0$, as a function of time for the case of simultaneous flocculation and creaming are shown in Fig. 5. Initially, the PSD shifts to the right toward larger size particles (see PSD at $t=5 \mathrm{hr}$ ) due to flocculation of the smaller particles. Since the stability to flocculation increases exponentially with increasing particle size, the smaller particles flocculate and form relatively stable larger particles. The larger particles then cream out and the PSD shifts to the left toward the smaller size particles (see PSD at $t=10 \mathrm{hr}$ ). The creaming rate is proportional to the square of the particle's diameter, hence the

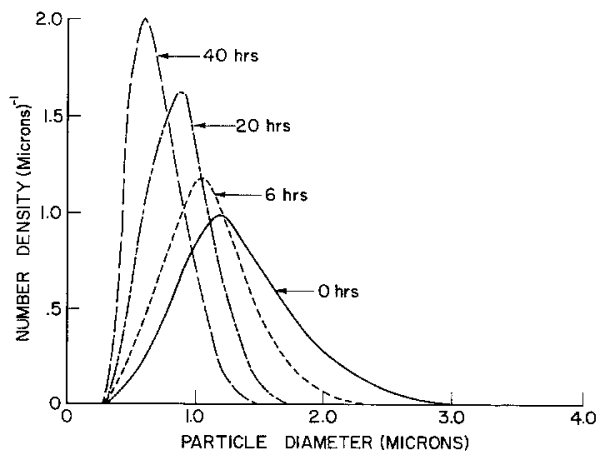

FIG. 3. Changes in the particle size distribution with time at $y=0$ for the case of creaming only. 
larger particles cream at a much faster rate than the smaller ones. This results in the shift of the PSD to the left. The PSD keeps becoming narrower (see PSD at $t=10,15$ $\mathrm{hr}$ ), because of slow flocculation due to the low concentrations of smaller particles. These cyclic changes or shifts in particle size distributions would not have been realized without including both flocculation and creaming in the solution to the general dynamic equations.

Variations in local particle size distributions are presented in the following figures. Total particle concentration varies in the direction of the gravitational field (Fig. 2). The particle size distribution also varies in the direction of the gravitational field, $y$. The PSDs corresponding to $y=0$ and $y \geq 10$ at time $t=5 \mathrm{hr}$ are shown in Fig. 6. The PSD remains the same in the region $y \geq 10$, and in the region $0 \leq y \leq 10$, the PSDs fall in between these two curves. Similar plots for time, $t=10 \mathrm{hr}$, are presented in Fig. 7. In this case, the PSD remains the same in the region $y \geq 30 \mathrm{~cm}$. The variations of PSD are presented in Fig. 8 for time, $t=20 \mathrm{hr}$, when only creaming is occurring. The larger particles cream out from the lower part of the column, which results in an increase in

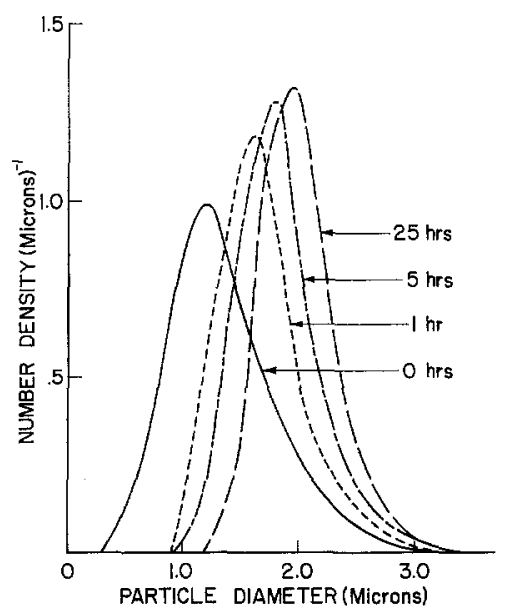

FIG. 4. Changes in the particle size distribution with time at $0 \leq y \leq L$ for the case of flocculation only.

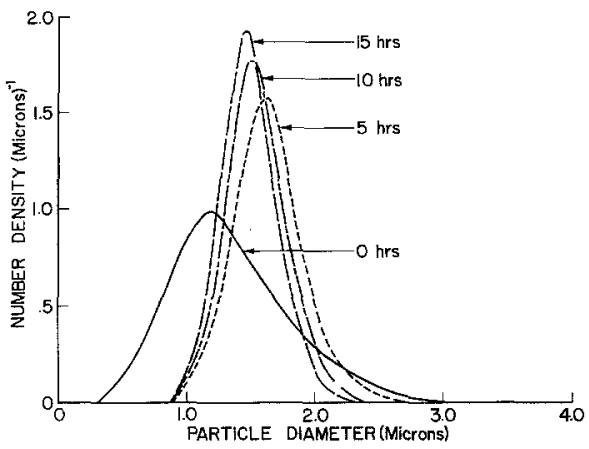

FIG. 5. Changes in the particle size distribution with time at $y=0$ for the case of simultaneous flocculation and creaming with $\psi=10 \mathrm{mV}$.

the relative concentration of smaller particles at the lower end (and larger particles at the upper end). One observes from Figs. 6,7 , and 8 that the particles at the bottom of the column, $y=0$, are smaller and narrowly distributed while at the top of the column the particles are larger and the distribution is less peaked (leptokurtic).

The behavior of this same emulsion had been predicted for various degrees of stabilization, i.e., for various values of surface potential. The PSDs at various times for a surface potential, $\Psi$, of $8 \mathrm{mV}$ are plotted in Fig. 9. As seen in this figure, initially the particles are small and the flocculation is significant, resulting in a very rapid PSD shift to the right. Once the particles are large



Frg. 6. Particle size distribution at various positions after $5 \mathrm{hr}$ for the case of simultaneous flocculation and creaming. 




FIG. 7. Particle size distribution at various positions after $\mathbf{1 0 ~ h r}$ for the case of simultaneous flocculation and creaming.

enough to cream faster and become relatively stable to flocculation, the PSD shift to the left results. The PSD continues to shift slowly further to the left with time, due to creaming of larger particles. The results for another case, that of $\Psi=9 \mathrm{mV}$, are plotted in Fig. 10. Initially, the particles flocculate and form larger particles. However, unlike the previous case, the particles do not become large enough to cream faster and shift the PSD to the left very rapidly. The results for yet another case, that of $\Psi=12 \mathrm{mV}$, are plotted in Fig. 11. In this case, the $\zeta$ potential is high enough to keep the particles stable to flocculation, and, as a result, mainly creaming occurs and the PSD shifts only to the left.

The cyclic changes in the particle size distribution are a function of the emulsion stability to flocculation. If the particles are very

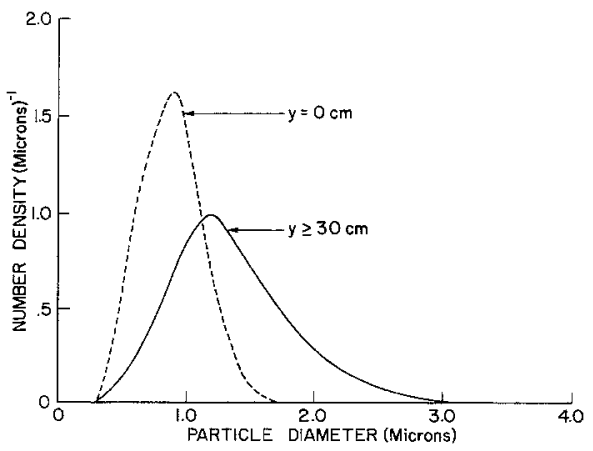

FIG. 8. Particle size distribution at various positions after $20 \mathrm{hr}$ for the case of creaming only. stable, the PSD shifts mostly to the left due to creaming and if particles are very unstable to flocculation, the PSD shifts mostly to the right. If flocculation and creaming are equally significant, a reverse shift in PSD takes place.

Trends in the changes of the concentration profiles of individual particles $\left(N_{i}\right.$ vs $y$ ) will be quite different from the trends for the total particle concentration, Fig. 2. The concentration profiles of particles of two representative sizes, 1.2 and $2.4 \mu \mathrm{m}$ in diameter, are plotted in Figs. 12 and 13. Figure 12 shows the two limiting cases: (1) only flocculation and (2) only creaming. One notes that the concentration of particles of any size will be spatially uniform for the case of flocculation without creaming. However, for the case of creaming without flocculation, the concentration of any size particle will increase in the direction of creaming.

It is quite interesting to observe the trends in concentration profiles for the case of simultaneous flocculation and creaming as presented in Fig. 13. Concentration profiles of smaller particles (e.g., $1.2 \mu \mathrm{m}$ ) are quite different from that of larger particles (e.g., $2.4 \mu \mathrm{m})$. Larger particles are primarily creaming, therefore, the concentration increases in the direction of creaming. In the case of the smaller $1.2-\mu \mathrm{m}$ diameter particles, the concentration decreases in the

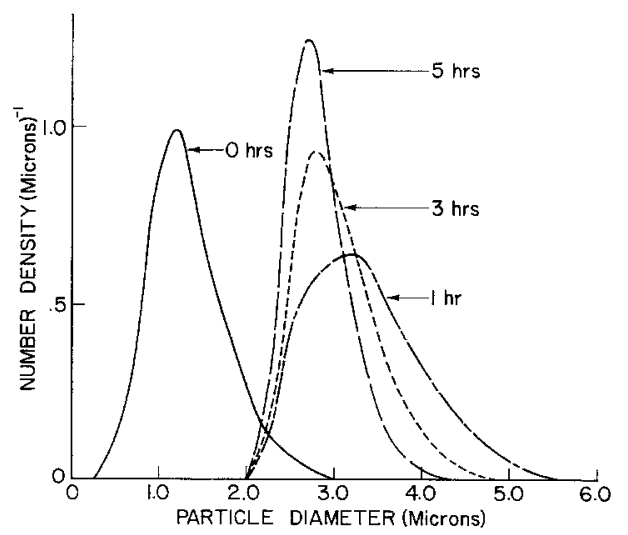

FIG. 9. Changes in the particle size distribution with time at $y=0$ for the case of simultaneous flocculation and creaming with $\psi=8 \mathrm{mV}$. 




FIG. 10. Changes in the particle size distribution with time at $y=0$ for the case of simultaneous flocculation and creaming with $\psi=9 \mathrm{mV}$.

direction of creaming (see $t=5 \mathrm{hr}$ ). Recalling Fig. 2, the total particle concentration at about $0.5 \mathrm{~cm}$ in height at $t=5 \mathrm{hr}$ is relatively low, therefore the net loss of $1.2-\mu \mathrm{m}$ particles by flocculation will be slow and since the particles are small, creaming is also negligible. In the region $y \geq 3$, the particle concentration is relatively high and the particles are disappearing by Brownian and sedimentation-flocculation. The net result is the concentration profiles shown in Fig. 13 (see $t=5,7$, and $10 \mathrm{hr}$ ). After about $15 \mathrm{hr}$ the particles in the region $y \leq 0.75 \mathrm{~cm}$ are slowly creaming and in the region $y \geq 2$ are slowly flocculating, resulting in a peak in concentration profile at about $1.0 \mathrm{~cm}$ as shown in Fig. $13(t=15 \mathrm{hr})$. Also in the region $y \geq 3 \mathrm{~cm}$, after a certain period of time the concentration of particles increases with time (see $t=15$ and $25 \mathrm{hr}$ ). Concentration profiles of smaller particles are qualitatively unpredictable unlike concentration profiles of any size in the cases of only flocculation and only creaming. The trends in concentration profiles of smaller particles can only be predicted by solving the general equation.

Another important feature in these various PSD curves is the widening and narrowing of the distributions at various aging times. Generally, in a creaming emulsion, the particle distribution slowly narrows to the left, due to creaming of larger particles. This can

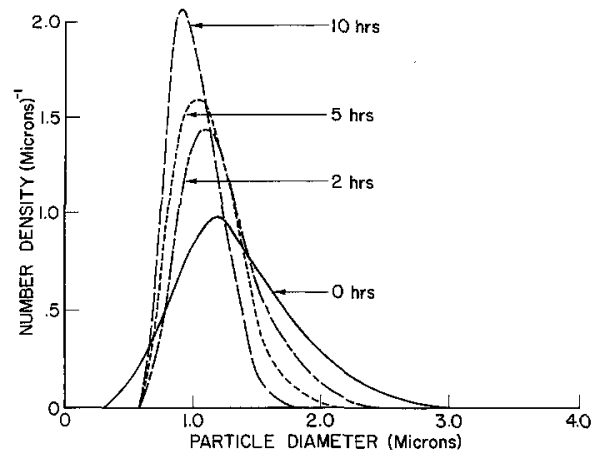

Frg. 11. Changes in the particle size distribution with time at $y=0$ for the case of simultaneous flocculation and creaming with $\psi=12 \mathrm{mV}$.

be seen in Fig. 4. However, in the case of flocculation and creaming, the PSD can widen, narrow down, or do both one after the other. In the case of $\Psi=10 \mathrm{mV}$, the PSD initially widens slightly and then narrows rapidly (Fig. S). In the case of $\Psi=12$ $\mathrm{mV}$, the PSD only narrows with aging time (Fig. 11). In this case, the surface potential and particle sizes are such that the lower half $(D \leq 1.0 \mu \mathrm{m})$ of the distribution nar-

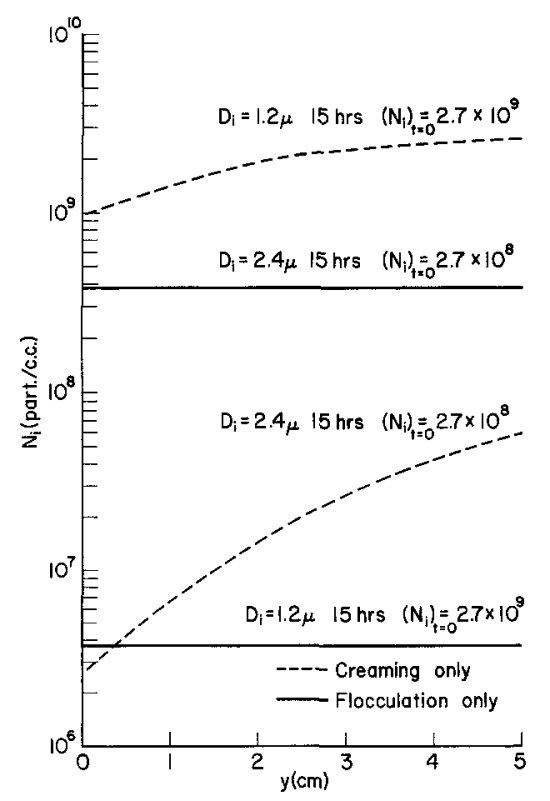

FIG. 12. Concentration of particles of specific size, $N_{i}$, as a function of position, $y$, for the cases of flocculation and creaming only. 


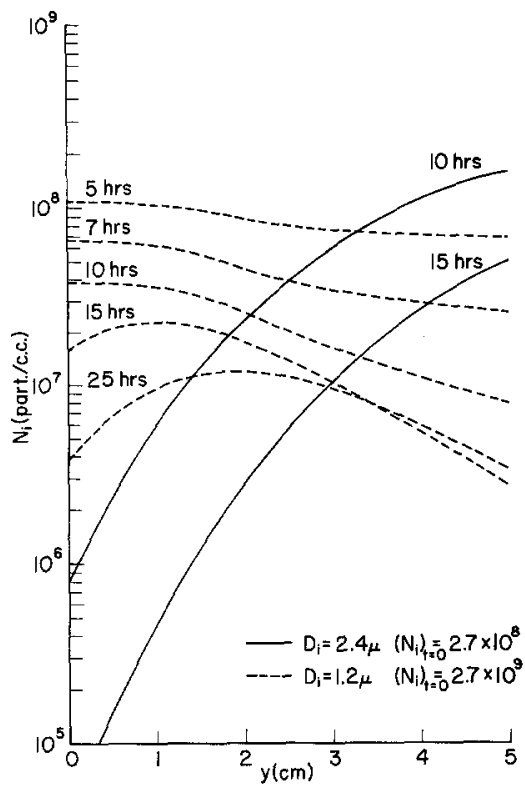

FIG. 13. Concentration of particles of specific size, $N_{i}$, as a function of position, $y$, for the case of simultaneous flocculation and creaming.

rows to the right due to flocculation, and the upper half $(D \geq 1.0 \mu \mathrm{m})$ of the distribution narrows to the left due to creaming (see Fig. 11). Similarly, one can predict the changes that take place in various types of emulsions stabilized to various extents.

\section{SUMMARY}

Flocculation and creaming occur simultaneously in many practical emulsions, such as demulsification and emulsion breaking. A model has been developed to predict or simulate the behavior of these types of emulsions. A population balance was used to generate a set of partial differential equations which describe the emulsion stability with simultaneous flocculation and creaming. Numerical solutions describe the changes in particle concentration and size distribution as a function of time and position. The behavior of various types of emulsions has been predicted by using the model. In a flocculating emulsion, the PSD shifts to the right toward larger particle sizes, whereas in the case of creaming it only shifts to the left toward the smaller particle sizes. In the case of simultaneous flocculation and creaming, the PSD initially shifts to the right and then reverses and shifts to the left.

In the following paper the experimental studies on simultaneous flocculation and creaming will be described and compared with the theoretical model and trends developed in this paper.

\section{REFERENCES}

1. Schall, E. C., Science News 1(4), \#25/26, 388 (1973).

2. Li, M. K., and Fogler, H. S., J. Fluid Mech. 88(3), 499 (1978).

3. Shah, D. O., and Schechter, R. S., (Eds.), "Improved Oil Recovery by Surfactant and Polymer Flooding." Academic Press, New York, 1977.

4. Wasan, D. T., Shah, S. M., Aderangi, N., Chan, M. S., and McNamara, J. J., Soc. Petrol. Eng. J. 18(6), 409 (1978).

5. Friedlander, S. K., "Smoke, Dust and Haze. Fundamentals of Aerosol Behavior." Wiley, New York, 1977.

6. Rasool, S. I., (Ed.), "Chemistry of the Lower Atmosphere." Chap. 3. Plenum, New York, 1973.

7. Okuyama, K., Kousaka, Y., and Yoschida, T., J. Chem. Eng. Japan 9(2), 140 (1976).

8. Saffman, P. F., and Turner, J. S., J. Fluid Mech. 1, 16 (1956).

9. Greenfield, M. A., Koontz, R. L., and Hausknecht, D. F., J. Colloid Interface Sci. 35(1), 102 (1971).

10. Kruyt, H. R., "Colloid Science,"' Vol. 1, Chap. 7. Elsevier, Amsterdam, 1952.

11. Ramkrishna, D., Chem. Eng. Sci. 26, 1136 (1971).

12. Reddy, S. R., Ph.D. Thesis, University of Michigan, 1981 .

13. Gelbard, F., Seinfeld, J. H., and Tambour, Y., J. Colloid Interface Sci. 76(2), 541 (1980).

14. Verwey, E. J. W., and Overbeck, J. Th. G., "Theory of the Stability of Lyophobic Colloids." Elsevier, Amsterdam, 1948.

15. Ho, N. R. H., and Higuchi, W. I., J. Pharm. Sci. 57, 436 (1968).

16. Honig, E. P., Roebersen, G. J., and Wiersma, P. H., J. Colloid Interface Sci. 36(1), 97 (1971).

17. Carnahan, B., Luther, A., and Wilkes, J. O., “Applied Numerical Methods," Chap. 7. Wiley, New York, 1969.

18. Lyklema, J., Advan. Colloid Interface Sci. 2, 65 (1968).

19. Suzuki, A., Ho, N. F. H., and Higuchi, W. I., J. Colloid Interface Sci. 29(3), 552 (1969). 